\title{
Microphase Ordering in Melts of Randomly Grafted Copolymers
}

\section{Citation}

Qi, Shuyan, Arup K. Chakraborty, Hao Wang, Amy A. Lefebvre, Nitash P. Balsara, Eugene I. Shakhnovich, Maria Xenidou, and Nikos Hadjichristidis. 1999. "Microphase Ordering in Melts of Randomly Grafted Copolymers." Physical Review Letters 82 (14): 2896-99. https:// doi.org/10.1103/physrevlett.82.2896.

\section{Permanent link}

http://nrs.harvard.edu/urn-3:HUL.InstRepos:41417275

\section{Terms of Use}

This article was downloaded from Harvard University's DASH repository, and is made available under the terms and conditions applicable to Other Posted Material, as set forth at http:// nrs.harvard.edu/urn-3:HUL.InstRepos:dash.current.terms-of-use\#LAA

\section{Share Your Story}

The Harvard community has made this article openly available.

Please share how this access benefits you. Submit a story.

Accessibility 


\title{
Microphase Ordering in Melts of Randomly Grafted Copolymers
}

\author{
Shuyan Qi ${ }^{1}$ Arup K. Chakraborty, ${ }^{1, *}$ Hao Wang, ${ }^{2}$ Amy A. Lefebvre, ${ }^{2}$ Nitash P. Balsara, ${ }^{2, *}$ \\ Eugene I. Shakhnovich, ${ }^{3}$ Maria Xenidou, ${ }^{4}$ and Nikos Hadjichristidis ${ }^{4}$ \\ ${ }^{1}$ Departments of Chemical Engineering and Chemistry, University of California, Berkeley, California 94720 \\ ${ }^{2}$ Department of Chemical Engineering, Chemistry, and Materials Science, Polytechnic University, Brooklyn, New York 11201 \\ ${ }^{3}$ Department of Chemistry and Chemical Biology, Harvard University, Cambridge, Massachusetts 02139 \\ ${ }^{4}$ Department of Chemistry, University of Athens, Panepistiomiopolis, Athens, Greece
}

(Received 28 July 1998)

\begin{abstract}
Using optical birefringence, small-angle neutron scattering, and field-theoretic methods, we study the effects of frustrating quenched randomness and connectivity on microphase ordering in copolymer melts. Our results show that randomly grafted copolymers are good model systems to examine these effects, and we find that these materials exhibit behavior different from that observed heretofore for other types of molten polymers. [S0031-9007(99)08822-5]
\end{abstract}

PACS numbers: 61.41.+e, 61.12.Ex

Mixtures of incompatible homopolymers form distinct macroscopic phases when cooled below a certain temperature. In contrast, due to frustration presented by chain connectivity, molten copolymers comprised of incompatible segments form ordered microphases when cooled below the phase transition temperature. The physics of microphase ordering in copolymers with ordered sequence distributions (e.g., diblock copolymers) has been studied extensively, e.g., [1], and many exotic microstructures with potential applications have been observed.

Recent years have witnessed a surge of interest in copolymers with disordered sequence distributions. A simple example is provided by connecting two types of segments to form linear chains with disordered sequences (a linear random copolymer or LRC). The disordered sequence distribution is quenched and leads to frustrations not present in copolymers with ordered sequence distributions. Fundamental interest in systems with quenched disorder and the relevance of LRCs as models to study protein folding have motivated many theoretical studies, e.g., [2]. The effects of frustrating quenched randomness and chain connectivity on microphase ordering in LRCs have been considered by theorists, e.g., [3]. Theorists have also examined the disordered molten state of polymers with quenched randomness and branched architectures, e.g., [46]. However, experiments probing microphase ordering in copolymers with disordered sequences have not been conducted, largely because of the difficulty of synthesizing LRCs with controlled sequence statistics.

We consider a class of copolymers that embodies competing interactions and frustrating sequence disorder that can be synthesized with well-characterized sequence statistics. Specifically, we have a homopolymer backbone (A-type segments) of length $N$ onto which are grafted $p$ branches ( $B$-type segments) of length $M$. The branch points are randomly located on the backbone and cannot anneal after synthesis is complete. Each chain in a melt of these copolymers has a different sequence of branch points. We refer to these materials as randomly grafted copolymers (or RGCs). An RGC was synthesized by a combination of anionic polymerization and selective silane coupling [7]. Microphase ordering in this wellcharacterized RGC melt wherein the branch point fluctuations are short range correlated with a mean value of $p / N$ is then studied by optical birefringence and small-angle neutron scattering (SANS). We also develop a field-theoretic model for describing microphase ordering in such RGCs. Our analysis shows that as the system is cooled below the microphase transition temperature, the length scale on which the system orders is first strongly temperature dependent and then becomes essentially temperature independent. In the latter region, the theoretical analysis is in excellent agreement with our SANS data. We discuss the physical origin of the observed behavior which is different from that predicted for LRCs and that observed for diblock copolymers.

Consider $N_{p}$ chains, with $\mathbf{r}_{i}(n)$ being the spatial location of the $n$th segment on the backbone of the $i$ th chain, and let $\mathbf{r}_{i j}(m)$ be the spatial location of the $m$ th segment of the $j$ th branch on the $i$ th chain. Let the set $\left\{n_{i j}\right\}$ represent the quenched locations of the branch points. The partition function for a particular realization of branch point locations can be written as

$$
\begin{aligned}
Z\left[n_{i j}\right]= & \iint D \rho_{A}(r) \iint D \rho_{B}(r) \\
& \times \exp \left\{S\left[\rho_{A}(r) ; \rho_{B}(r) ; n_{i j}\right]\right. \\
& \left.-E\left[\rho_{A}(r) ; \rho_{B}(r)\right]\right\},
\end{aligned}
$$

where $\rho_{A}$ and $\rho_{B}$ are coarse-grained macroscopic fields corresponding to local volume fractions. The energy $E$ is

$$
E=\frac{1}{2} \int d r\left\{-2 \chi m^{2}(r)+c^{2}[\nabla m(r)]^{2}\right\},
$$

where $c^{2}$ is the surface tension, $\chi$ is the Flory parameter, $m(\mathbf{r})=\zeta_{B} \rho_{A}(\mathbf{r})-\zeta_{A} \rho_{B}(\mathbf{r})$, and $\zeta_{A}$ and $\zeta_{B}$ are the average volume fractions of $A$ - and $B$-type segments. The 
entropy $S$ is given by

$$
\begin{aligned}
\ln & \prod_{i=1}^{N_{p}} \iint D r_{i}(n) \prod \iint D r_{i j}(m) \exp \left[-\frac{3}{2 l} \sum_{i=1}^{N_{p}} \int d n\left(\frac{d r_{i}(n)}{d n}\right)^{2}-\frac{3}{2 l} \sum_{j} \sum_{i=1}^{N_{p}} \int d m\left(\frac{d r_{i j}(m)}{d m}\right)^{2}\right] \\
& \times \prod_{i=1}^{N_{p}} \prod_{j} \delta\left(r_{i j}(0)-r_{i}\left(n_{i j}\right)\right) \delta\left[\rho_{A}(r)-\sum_{i=1}^{N_{p}} \int d n \delta\left(r_{i}(n)-r\right)\right] \delta\left[\rho_{B}(r)-\sum_{i=1}^{N_{p}} \sum_{j} \int d m \delta\left(r_{i j}(m)-r\right)\right],
\end{aligned}
$$

where $l$ is the statistical segment length of $A$ - and $B$-type segments, and we have subsumed appropriate factors of volume in the definitions of the $\delta$ functions.

We now need to perform a quenched average over the branch point locations in order to obtain the free energy corresponding to a particular set of macroscopic density fields. Using the replica method [8] to compute the quenched average, and introducing the fields $\gamma(\mathbf{r})$ and $\phi(\mathbf{r})$ conjugate to the single chain fields, $\rho^{A}$ and $\rho^{B}$, obtains

$$
\begin{aligned}
\left\langle R^{n}\right\rangle= & \prod_{\alpha} \iint D \phi_{\alpha}(r) \iint D \gamma_{\alpha}(r) \exp \left\{i \sum_{\alpha} \int d r\left[\gamma_{\alpha}(r) \rho_{\alpha}^{A}(r)+\phi_{\alpha}(r) \rho_{\alpha}^{B}(r)\right]\right\} \\
& \times\left\langle\prod_{\alpha} \iint D r_{\alpha}(n) \prod \iint D r_{j \alpha}(m) P\left[r_{j \alpha}(m)\right] P\left[r_{\alpha}(n)\right] \prod_{j} \delta\left(r_{j \alpha}(0)-r_{\alpha}\left(n_{j}\right)\right)\right\rangle
\end{aligned}
$$

with the averaged entropy $\langle S\rangle=\lim _{n \rightarrow 0}\left(\left\langle R^{n}\right\rangle-1\right) / n$. We have taken $N_{p}(N+p M) / V=1$, and the angular bracket denotes the quenched average; $P\left[r_{\alpha}(n)\right]=P_{0}\left[r_{\alpha}(n)\right] \exp \left[-i \int d n \gamma_{\alpha}\left(r_{\alpha}(n)\right)\right], \quad P\left[r_{j \alpha}(m)\right]=$ $P_{0}\left[r_{j \alpha}(m)\right] \exp \left[-i \sum_{j} \int d m \phi_{\alpha}\left(r_{j \alpha}(m)\right)\right] . \quad P_{0}$ are the Gaussian functions in preceding equations that enforce connectivity, and $i=\sqrt{-1}$. Although each chain has a different sequence, since the distribution function describing the fluctuations in branch point locations is the same we use the symbol $n_{j}$ in Eq. (4).

We now expand the functionals $P$ in powers of the conjugate fields up to fourth order, with the bare propagators being Gaussian $\left(P_{0}\right)$. Carrying out the resulting integrals, performing the average over the distribution of $n_{j}$, and evaluating the functional integrals over the conjugate fields using saddle points we find the replica symmetric solution for the entropy to be

$$
\begin{aligned}
\langle S\rangle= & -\frac{1}{2} \int d q \vec{\rho} \underline{\underline{W}} \vec{\rho}-\frac{1}{8}\left[\int d q \vec{\rho} \underline{\underline{W}} \overrightarrow{\boldsymbol{\rho}}\right]^{2}-\sum_{i, j, k=A \vee B} \int d q_{1} \int d q_{2} \rho_{i}\left(q_{1}\right) \rho_{j}\left(q_{2}\right) \rho_{k}\left(-q_{1}-q_{2}\right) \Gamma_{i j k}^{(3)}\left(q_{1}, q_{2}, q_{3}\right) \\
& -\frac{1}{8} \int d q_{1} \int d q_{2} \vec{\rho}^{T}\left(q_{1}\right) \underline{W}\left(q_{1}\right)\left[\left\langle\underline{M_{0}}\left(q_{1}\right) \underline{W}\left(q_{1}\right) \vec{\rho}^{T}\left(q_{1}\right) \vec{\rho}\left(q_{2}\right) \underline{W}\left(q_{2}\right) \underline{M_{0}}\left(q_{2}\right)\right\rangle-\vec{\rho}^{T}\left(q_{1}\right) \vec{\rho}\left(q_{2}\right)\right] \underline{W}\left(q_{2}\right) \vec{\rho}\left(q_{2}\right) \\
& -\sum_{i, j, k, l=A \vee B} \int d q_{1} \int d q_{2} \int d q_{3} \rho_{i}\left(q_{1}\right) \rho_{j}\left(q_{2}\right) \rho_{k}\left(q_{3}\right) \rho_{l}\left(-q_{1}-q_{2}-q_{3}\right) \Gamma_{i j k l}^{(4)}\left(q_{1}, q_{2}, q_{3}\right),
\end{aligned}
$$

where $\left\langle\underline{M_{0}}\right\rangle=\underline{W}^{-1}, \vec{\rho}^{T}=\left(\rho^{A}, \rho^{B}\right)$, and underlined symbols are matrices.

Combining Eq. (5) with Eq. (2) obtains the free energy functional that we seek. Detailed derivations of the mathematical formulas for $M_{0}, \Gamma^{(3)}$, and $\Gamma^{(4)}$ will be presented in a forthcoming longer account [9]. Here, for brevity and in order to see the essential issues clearly, we remark on their mathematical forms before presenting detailed results. The quadratic term $\left(F_{2}\right)$ in our free energy functional contains terms proportional to $q^{2}$ and $1 / q^{2}$. The physical origin of the Coulombic term in $F_{2}$ is the stoichiometric constraint that forces the branches to be connected to the backbone at specific points. $F_{2}(q)$ depends upon temperature but exhibits a minimum with a temperature independent value of $q$ at the minimum. In an earlier effort [5], a free energy functional for molten RGCs was obtained up to quadratic order (identical to $F_{2}$ ), and the structure factor in the disordered phase was studied as a function of various parameters. However, as mentioned in passing in [5] and as we shall see, the quartic terms play an important role in determining the physics of microphase ordering. The fourth term in Eq. (5), $F_{41}(q)$, originates from the quenched fluctuations in branch point locations. It is a temperature dependent monotonic function that decays sharply with $q$ at relatively small values of $q$. The last term in Eq. (5) $\left(F_{42}\right)$ contains contributions from the mean values of the quenched disorder. It resembles $F_{2}(q)$ with the minimum shifted to higher values of $q$.

In this first attempt to understand microphase ordering in incompressible $\left(1=\rho_{A}+\rho_{B}\right)$ RGC melts, we consider a lamellar microstructure. Thus, we take $\rho_{A}(\mathbf{r})=$ $D \cos (\mathbf{q} \cdot \mathbf{r})+\zeta_{A}$. Our results are obtained by minimizing the free energy with respect to $D$ and $|q|$.

Figure 1 shows our prediction of the optimal wave vector as a function of $\chi M$ for $N=2138, M=285$, and $p=16$ (the reason for these choices will be made clear shortly). At high temperature (low $\chi M$ ) we have a disordered phase, and $q^{*}$ is small and invariant with 


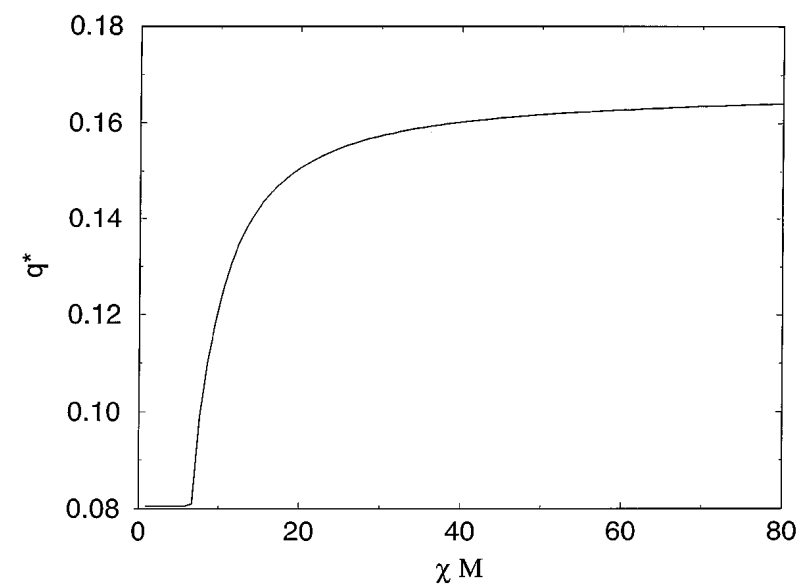

FIG. 1. Optimal wave vector (nondimensionalized by $l$ ) as a function of $\chi M$.

temperature. Immediately below the microphase separation transition (MST) $q^{*}$ increases strongly with temperature, a feature reminiscent of earlier predictions for LRCs [3]. At lower temperatures yet, $q^{*}$ becomes essentially temperature independent. The range of temperature over which $q^{*}$ varies strongly with temperature depends upon $M$ and $p$. For LRCs, a continuous variation of $q^{*}$ with temperature is predicted [3]. For diblock copolymers, $q^{*}$ decreases with temperature upon ordering largely due to chain stretching [10].

The reason for the unusual variation of $q^{*}$ with temperature for RGCs is the following. As in [5], in the disordered state $D^{2}=0$ and $q^{*} \sim(N+p M)^{-1 / 2}$. Below the MST, the system begins to order on smaller scales. Immediately below the MST, the scale on which the system orders is still much larger than the branch length, and so RGCs approach the limit of LRCs. Because of uncorrelated fluctuations in the branch point locations, there is no natural length scale on scales much larger than the branch length. As for LRCs [3], this makes $q^{*}$ a strong function of temperature because as temperature decreases the entropic contribution to the free energy continuously becomes less important, and the system orders on progressively smaller scales due to the energetic driving force. Mathematically, $q^{*}$ is determined by the interplay of $F_{41}$ and $F_{2}$ (since $F_{41} \gg F_{42}$ at this stage), and it increases sharply as temperature decreases because of the form of $F_{41}$ discussed earlier. For LRCs, this trend continues, and $q^{*}$ approaches monomeric scales. However, for RGCs, when $q^{*}$ corresponds to scales commensurate with the branch length, a natural length scale (the branch length) emerges. Thus, once the temperature is low enough that $q^{*}$ corresponds to the length scale of the branches, it becomes essentially temperature independent. Now $F_{41} \ll F_{42}$, and the mean value of the quenched randomness is more important than the strength of the fluctuations. We caution that deep in the ordered region $(\chi M \gg 10)$ our analysis is inappropriate because of strong segregation effects and chain stretching [10].
We prepared RGCs with a polybutadiene (PB) backbone and polystyrene (PS) branches. We first synthesize the PB backbone using standard anionic polymerization. The resultant polymer contained 91\% $1-4$ units and 9\% $1-$ 2 units [11]. This polymer is then reacted with $\mathrm{HSiMe}_{2} \mathrm{Cl}$ in the presence of a $\mathrm{Pt}$ catalyst which selectively silates the pendant $\mathrm{C}=\mathrm{C}$ bond in the $1-2$ units. The silated PB is then reacted with living polystyryllithium, which is polymerized in a separate reactor by conventional anionic polymerization, to give the RGC. The backbone, the branches, and the final RGC were characterized by size exclusion chromatography, light scattering, and NMR spectroscopy [7]. We can prepare polymers with different values of $p, N$, and $M$ by adjusting the molecular weights of the PS and PB precursors and the concentration of the silane coupling agent. As a first step, we have prepared a RGC with $N=2138, M=285$, and $p=16$. (The experimental parameters $\chi, N, M$, and $l$ are based on a monomer reference volume of $100 \AA^{3}$.) The reason for the choice of parameters in Fig. 1 should now be clear.

Optical birefringence and SANS experiments were conducted on a $1 \mathrm{~mm}$ thick melt of the RGC held between optical flats. The experiments were conducted over a temperature range of 110 and $180{ }^{\circ} \mathrm{C}$ (above the glass transition temperatures of PS and PB). The apparatus used for the optical experiments is described in [12]. The sample was held between crossed polarizers in a temperature controlled stage and the total transmitted light in the range $0<q<2.56 \mu \mathrm{m}^{-1}(q=4 \pi \sin (\theta / 2) / \lambda, \theta$ is the scattering angle, and the wavelength of incident light, $\lambda=$ $0.632 \mu \mathrm{m}$ ) was focused onto a photodetector using a lens. The temperature dependence of the measured optical signal is shown as an inset in Fig. 2. In previous studies of diblock copolymers [12], it was shown that the orderdisorder transition is announced by a discontinuous drop in the birefringence signal to less than 1 millivolt. The birefringence signal from the RGC melt is finite and comparable in magnitude to that obtained for ordered diblock copolymers [12] over the entire temperature range that we have studied. This is indicative of an ordered phase; i.e., for these values of $N, M, p$, and density we are unable to access the MST. However, extrapolation of the birefringence data suggests that the MST occurs at $\chi M \sim O(10)$. This is consistent with the prediction in Fig. 1.

SANS experiments on the RGC melt were conducted on the NG5 beam line at the NIST in Maryland [13]. The measured scattering intensity was corrected for background and empty cell scattering, detector sensitivity, incoherent scattering, and converted to absolute coherent scattering intensity, $I(q)$ ( $\lambda=1.2 \mathrm{~nm}$ for neutrons) [14]. We find the scattering profiles (shown at selected temperatures in Fig. 2) to be independent of thermal history. A single scattering peak is evident at all temperatures, and the coherent scattering intensity goes to zero (within experimental uncertainty) in both high and low $q$ limits. These features, coupled with finite birefringence signals, are classic signatures of microphase ordering in weakly 


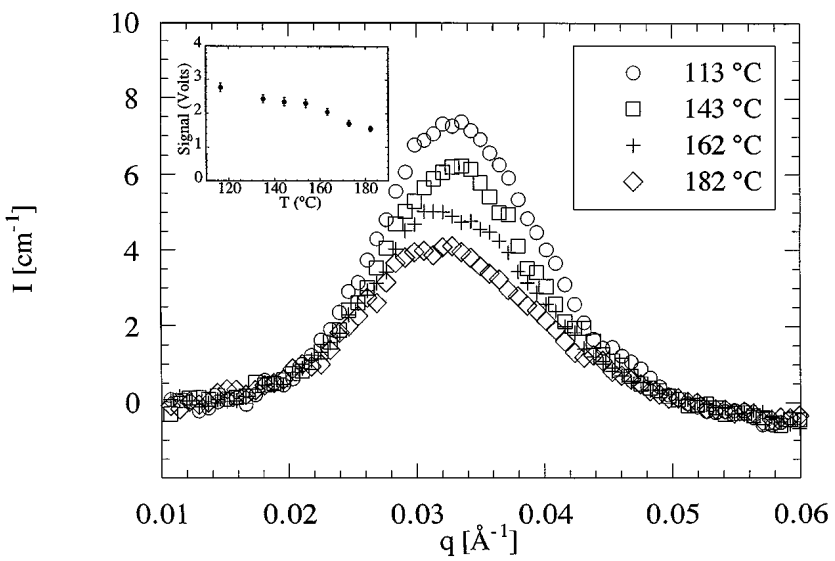

FIG. 2. Experimental data for a PS/PB RGC melt at selected temperatures in the ordered state. Main figure: SANS data; inset: optical birefringence.

ordered systems (e.g., [12]). However, the widths of the scattering peaks we obtain are considerably larger than those seen for ordered diblock copolymers [1]. This is clearly the effect of randomness in branch point location.

The order parameter in our theory is characterized by two parameters, $q^{*}$ and the scattering intensity at the peak $\left(I^{*} \propto|D|^{2}\right)$. Figure 3 compares the experimentally determined $I^{*}$ with theory. We assume that $\chi$ has the familiar temperature dependence $\chi=A+B / T$ and adjust the values of $A$ and $B$ to obtain the best fit to the experimental data. We find that $A$ is vanishingly small $(\sim 0.003)$, and $B=20$ obtains the best fit shown in Fig. 3. This value of $B$ is in agreement with that reported [15] for a PS/PB diblock copolymer $(B=19)$. According to Fig. 1, the corresponding values of $\chi M$ place the experimental data in a region close to the crossover from strongly temperature dependent to essentially temperature independent values of $q^{*}$. In this window, our theory indicates (using $l=5.02 \AA$ for PS [15]) that $q^{*}$ should lie between 0.027 and $0.028 \AA^{-1}$. For reference, we also note that the

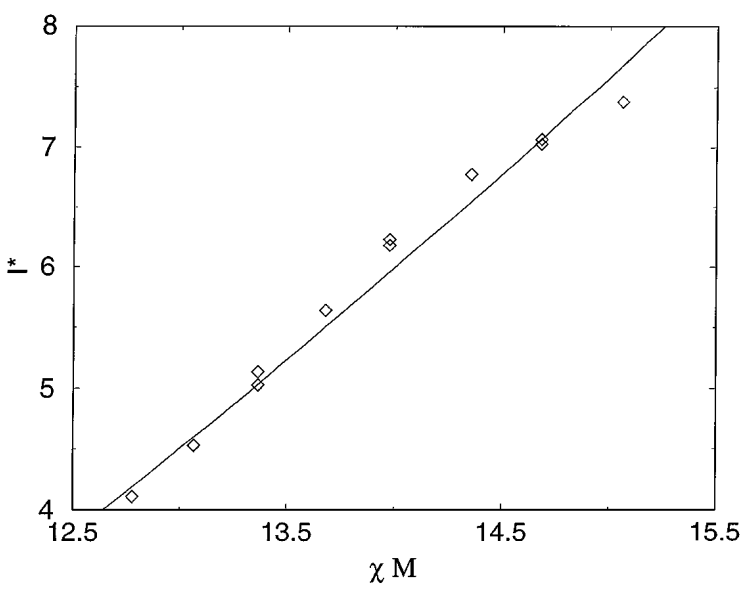

FIG. 3. Peak scattering intensity in SANS experiments as a function of $\chi M . \diamond$ symbols are measured values, and the curve represents the theoretical fit. asymptotic value of $q^{*}$ deep in the ordered region (Fig. 1) is $0.033 \AA^{-1}$. Within experimental uncertainties, the data in Fig. 2 show that $q^{*}$ is independent of temperature and equals $0.032 \pm 0.001 \AA^{-1}$. The agreement between theory and experiment is reasonable considering the experimental uncertainties in $\chi, M, N$, and $p$.

To summarize, we demonstrate that melts of randomly grafted copolymers are good model systems to study the effects of frustration due to quenched randomness on microphase ordering. In the region where we have experimental data, our theory and experiments are in agreement. Further work mapping out other regions of the phase diagram by varying $\chi, N, M$, and $p$ seems warranted.

We thank the polymer blends group and Center for Neutron Research at NIST for access to the NG5 beam line [13]. We acknowledge funding by NSF (DMR9457950) and U.S. DOE (BES).

*Corresponding authors.

[1] L. Leibler, Macromolecules 13, 1602 (1980); F. S. Bates and G. H. Fredrickson, Annu. Rev. Phys. Chem. 41, 525 (1990).

[2] A. Sali, E. I. Shakhnovich, and M. Karplus, Nature (London) 369, 248 (1994); H. Frauenfelder and P. G. Wolynes, Phys. Today 47, No. 2, 57 (1994); S. Srebnik, A. K. Chakraborty, and E. I. Shakhnovich, Phys. Rev. Lett. 77, 3157 (1996).

[3] E.I. Shakhnovich and A.M. Gutin, J. Phys. (Paris) 50, 1843 (1989); G. H. Fredrickson, S. T. Milner, and L. Leibler, Macromolecules 25, 6341 (1992).

[4] A.M. Gutin, A. Yu. Grosberg, and E.I. Shakhnovich, Macromolecules 26, 3598 (1993).

[5] A. Shinozaki, D. Jasnow, and A.C. Balazs, Macromolecules 27, 2496 (1994).

[6] A. Werner and G. H. Fredrickson, J. Polym. Sci. B 35, 849 (1997).

[7] M. Xenidou and N. Hadjichristidis, Macromolecules 31, 5690 (1998).

[8] S.F. Edwards and P.W. Anderson, J. Phys. F 5, 965 (1975).

[9] S. Qi et al. (to be published).

[10] A. Mayes and M. Olvera de la Cruz, Macromolecules 24, 3975 (1991); H. Tang and K. Freed, J. Chem. Phys. 96, 8621 (1992); J. L. Barrat and G. H. Fredrickson, ibid. 95, 1281 (1991); M. Olvera de la Cruz, Phys. Rev. Lett. 67, 85 (1991); K. Almdal et al., ibid. 65, 1112 (1990).

[11] M. Morton and L. J. Fetters, Rubber Chem. Technol. 48, 359 (1975).

[12] N.P. Balsara et al., Macromolecules 25, 6072 (1992); C. C. Lin et al., Macromolecules 27, 7769 (1994).

[13] Identification of equipment or materials does not imply recommendation by the NIST.

[14] N. P. Balsara et al., J. Chem. Phys. 99, 10011 (1993).

[15] L.J. Fetters et al., Physical Properties of Polymers Handbook, edited by J.E. Mark (AIP Press, Woodbury, NY, 1996), Chap. 24. 